\title{
Impact of Baseline BMI upon the Success of Latina Participants Enrolled in a 6-Month Physical Activity Intervention
}

\author{
Sheri J. Hartman, ${ }^{1}$ Shira I. Dunsiger, ${ }^{2}$ Dori W. Pekmezi, ${ }^{3}$ Brooke Barbera, ${ }^{2}$ \\ Charles J. Neighbors, ${ }^{4}$ Becky Marquez, ${ }^{1}$ and Bess H. Marcus ${ }^{1}$ \\ ${ }^{1}$ Department of Family and Preventive Medicine, University of California, San Diego, 3855 Health Sciences Drive \\ No. 0901, La Jolla, CA 92093-0901, USA \\ ${ }^{2}$ Centers for Behavioral and Preventive Medicine, The Miriam Hospital and Warren Alpert Medical School of \\ Brown University, Providence, RI 02903, USA \\ ${ }^{3}$ Department of Health Behavior, University of Alabama at Birmingham, Brimingham, AL 35293, USA \\ ${ }^{4}$ The National Center on Addiction and Substance Abuse, Columbia University, New York, NY 10017, USA
}

Correspondence should be addressed to Sheri J. Hartman, sjhartman@ucsd.edu

Received 10 June 2011; Revised 21 September 2011; Accepted 11 October 2011

Academic Editor: Jack A. Yanovski

Copyright (C) 2011 Sheri J. Hartman et al. This is an open access article distributed under the Creative Commons Attribution License, which permits unrestricted use, distribution, and reproduction in any medium, provided the original work is properly cited.

\begin{abstract}
High rates of obesity in Latinas highlight the need to determine if physical activity interventions are equally effective across the body mass index (BMI) range. Thus, this study assessed how BMI impacts success of Spanish-speaking Latinas in a culturally and linguistically adapted theory-based physical activity intervention $(N=45)$. Longitudinal regression models tested the relationship between baseline BMI and outcomes. Overall, a trend for a negative association was found between baseline BMI and self-reported physical activity and theoretical constructs targeted by the intervention over time. For example, someone with a $25 \mathrm{~kg} / \mathrm{m}^{2}$ BMI would report, on average, 27.5 more minutes/week of activity compared to someone with a $30 \mathrm{~kg} / \mathrm{m}^{2} \mathrm{BMI}$ at followup. Furthermore, higher baseline BMI was significantly associated with lower self-efficacy, behavioral and cognitive processes of change, and family social support over time. These findings suggest that participants with higher BMI may need additional intervention to promote physical activity.
\end{abstract}

\section{Introduction}

Rates of obesity and related medical conditions (e.g., diabetes) are high in the Latino population, particularly among Latinas [1]. Lifestyle factors, such as inactivity, contribute to this public health crisis. Sedentary behaviors (i.e., television viewing) are often an integral part of family life in this community. For example, in past studies, Latina focus group participants reported frequently watching telenovelas in the evening with family, watching TV during meals, and using TV as babysitter and tool to learn English [2, 3]. On the other hand, finances, gender roles, and caretaking responsibilities can be major barriers to Latinas engaging in the recommended levels of physical activity ( 150 minutes of weekly moderate or vigorous physical activity). According to recent CDC data [4], 53.4\% of Latinos reported engaging in no leisure time activity, compared to $35.3 \%$ non-Hispanic Whites. Effective interventions are obviously needed, yet the high (and rising) rates of obesity in this group highlight the need to determine if existing physical activity interventions are equally effective across the body mass index (BMI) range.

While previous research has shown that higher BMI is associated with less physical activity $[5,6]$, there is limited evidence regarding the relationship between baseline BMI on subsequent change in a physical activity intervention. King and colleagues found that individuals with BMI $>31 \mathrm{~kg} / \mathrm{m}^{2}$ were engaging in, on average, far less than the recommended activity levels at every measurement point over a 2-year intervention. In contrast, participants with BMI $<31 \mathrm{~kg} / \mathrm{m}^{2}$ 
were, on average, exceeding the guidelines. While this study was conducted with predominantly White, non-Hispanic men and women, such findings emphasize the need to understand the role of BMI on adoption of physical activity and raises questions about whether general physical activity interventions will meet the needs of obese participants as well as nonobese participants. As prevalence rates of obesity among Latinas are much higher than non-Hispanic Whites, investigation into how BMI might specifically affect the success of Latinas in a physical activity intervention is needed and will help inform future efforts to better serve this population.

The Transtheoretical Model (TTM) [7] and the Social Cognitive Theory (SCT) [8] are two theoretical models frequently used in physical activity promotion. TTM states that individuals move through a series of stages (i.e., Precontemplation, Contemplation, Preparation, Action, and Maintenance) when starting and maintaining physical activity. As individuals move through the stages, TTM states that people weigh the pros and cons of becoming active. In addition, individuals use behavioral and cognitive processes or strategies to help them move through the stages. Behavioral processes refer to engaging in activities such as enlisting social support, rewarding yourself, and reminding yourself. Cognitive processes refer to activities such as increasing knowledge, comprehending benefits, and increasing healthy opportunities. Similarly the SCT states that there are behavioral and cognitive factors that influence individual's behaviors, such as social support and selfefficacy. Self-efficacy is one of the most central factors to behavior change for the SCT and refers to an individual's confidence about being physically active. Using a theoretical framework, such as SCT, is important for being able to properly implement and test an intervention. A review of physical activity interventions with Latinas found that only 6 out of the 12 interventions used any theoretical model, and the model that was most commonly used was the Social Cognitive Theory (Sharma, 2008 obesity reviews). Four out of those 6 studies used the SCT and all 4 studies that used SCT were successful in increasing physical activity, further supporting the need for theory-based interventions.

Thus, using data from the intervention arm of a trial of a six-month, culturally and linguistically adapted intervention for Latinas $(N=45)$ [9], that was developed and individually tailored based on TTM and SCT, the current study assessed whether baseline BMI predicted changes in minutes of at least moderate intensity physical activity and changes in associated SCT and TTM theoretical constructs (self-efficacy, cognitive and behavioral processes, and social support) targeted by the intervention over time. The two aims of the study were (1) to assess how BMI impacts Latinas' success in increasing physical activity and (2) to assess how BMI impacts success in changing theoretical constructs targeted by the intervention. We hypothesized that participants with lower BMIs would report greater increases in physical activity and greater changes to the theoretical constructs self-efficacy, cognitive and behavioral processes, and social support, in response to a theory-based physical activity intervention than women with higher BMIs.

\section{Method}

2.1. Design. Data are taken from a subsample of a larger parent study testing the efficacy of a culturally and linguistically adapted, individually tailored physical activity intervention $(N=93$; [9]). The physical activity intervention was designed to impact health behavior (minutes of physical activity) through changes in targeted TTM and SCT constructs (see Section 2.3). The current study focuses on the subgroup of Latina participants $(N=45)$ who received the theory-based physical activity intervention. We focused these analyses on the participants who received the intervention in order to test if the intervention was equally successful in increasing physical activity and changing the targeted theoretical constructs across the BMI range. Written informed consent was obtained, and the protocol was approved by the institutional review board of Brown University.

2.2. Participants. This sample was comprised of 45 Spanishspeaking women between 18 and 65 years old who selfidentified as Latina/Hispanic and who were engaging in less than two days of moderate or vigorous physical activity for 30 minutes or less each day. Participants were recruited through community-based organizations and Spanishlanguage newspapers and radio stations. Exclusion criteria included any serious medical condition that would make physical activity unsafe (history of coronary heart disease, diabetes, stroke, orthopedic problems), current or planned pregnancy, BMI above $40 \mathrm{~kg} / \mathrm{m}^{2}$, consuming three or more alcoholic drinks per day on five or more days per week, current suicidal ideation or psychosis, current clinical depression, hospitalization due to a psychiatric disorder in the past three years, and/or taking medication that may impair physical activity tolerance or performance (e.g., beta blockers).

2.3. Intervention. The physical activity intervention was based on the TTM [7] and SCT [8] and emphasized behavioral strategies for increasing activity levels, such as goal setting, self-monitoring, problem-solving barriers, increasing social support, and rewarding oneself for meeting physical activity goals. This six-month intervention consisted of monthly mailings of physical activity manuals that were matched to the participant's current TTM level of motivational readiness and individually tailored computer expert system feedback reports based on TTM and SCT constructs, including stages and processes of change, decisional balance, and self-efficacy [10-13]. To further encourage participants to use behavioral techniques pedometers and physical activity logs were provided, along with tip sheets on topics such as stretching. Details of the culturally and linguistically adapted physical activity intervention are described in detail elsewhere [9]. Delivery channel and intervention approach were informed by feedback from focus groups participants who indicated that this mail-based, tailored intervention was an appropriate, appealing delivery modality that addresses the preferences and needs expressed by Latinas [9]. 
2.4. Measures. At baseline, demographic information (age, education, income, race, ethnicity, language preference, nativity, marital status) was collected. At baseline and six months height and weight were measured. Height and weight were measured by the research staff at our facility using a digital Health-o-Meter medical scale that measured body weight to the quarter pound and a stadiometer to measure height to the quarter inch. The same scale and stadiometer were used for all participants and all assessments.

Physical activity was assessed at baseline, three months, and six months using the 7-day Physical Activity Recall (PAR). The PAR is an interviewer-administered instrument that provides an estimate of weekly minutes of physical activity $[14,15]$. The PAR uses multiple strategies for increasing accuracy of recall, such as breaking down the week into daily segments (i.e., morning, afternoon, evening) and asking about many types of activities, including time spent sleeping and in moderate, hard, and very hard activity. This measure has consistently demonstrated acceptable reliability, internal consistency, and congruent validity with other more objective measures of activity levels [16-19], as well as sensitivity to changes in moderate intensity physical activity over time [20,21].

Monthly, stages and processes of change and self-efficacy were measured through the mail and used to generate tailored intervention materials. The Stages of Change for Physical Activity (SCPA) [22] has demonstrated reliability (Kappa $=0.78$; intraclass correlation $r=0.84)$ and shown acceptable concurrent validity with measures of self-efficacy and current activity levels [22]. The Processes of Change (POC) [23] measures behavioral processes (i.e., substituting alternatives, enlisting social support, rewarding yourself, committing yourself, and reminding yourself), and cognitive processes (increasing knowledge, warning of risks, caring about consequence to others, comprehending benefits, and increasing healthy opportunities). The behavioral process subscale and the cognitive processes subscale each consist of 20 items that are scored on a 5-point Likert scale from 1 to 5 and then averaged to get a total subscale score. The Processes of Change has been shown to have a high internal consistency of 0.83 [23]. Self-Efficacy (SE) to become physically active across diverse contexts was measured with a 5-item instrument scored on a 5-point Likert scale from 1 to 5. Items are averaged to create a total score $($ alpha $=0.82)$ [23].

At baseline and six months social support was measured using the Social Support for Exercise (SSE) scale [24]. This instrument has three subscales: Family Participation, Family Rewards and Punishment, and Friends Participation. Items are scored on a 6-point Likert scale from 0 to 5 and summed. The three subscales have acceptable internal consistencies (alphas range 0.61 to 0.91 ) and demonstrate good criterion validity [24].

All of the measures were delivered in Spanish and were previously tested during a demonstration trial and vetted in individual interviews and focus groups.

2.5. Statistical Analyses. Descriptive statistics were calculated. Means and corresponding standard deviations are
Table 1: Demographic characteristics $(N=45)$.

\begin{tabular}{|c|c|}
\hline Characteristic & Intervention \\
\hline Born outside of continental U.S & $91 \%$ \\
\hline $\begin{array}{l}\text { Speak only Spanish or more Spanish than } \\
\text { English at home }\end{array}$ & $89 \%$ \\
\hline \multicolumn{2}{|l|}{$B M I$} \\
\hline Obese $(\mathrm{BMI} \geq 30)$ & $42 \%$ \\
\hline Overweight (BMI = 25-29.9) & $38 \%$ \\
\hline Normal (BMI =18.5-24.9) & $20 \%$ \\
\hline \multicolumn{2}{|l|}{ Educational level } \\
\hline High school graduate or less & $47 \%$ \\
\hline Some college/technical school & $22 \%$ \\
\hline College graduate or more & $31 \%$ \\
\hline \multicolumn{2}{|l|}{ Employment status* } \\
\hline Unemployed & $44 \%$ \\
\hline Full time & $31 \%$ \\
\hline Part time & $24 \%$ \\
\hline \multicolumn{2}{|l|}{ Yearly household income** } \\
\hline$<\$ 10,000$ & $22 \%$ \\
\hline$\geq \$ 10,000$ but $<\$ 20,000$ & $36 \%$ \\
\hline$\geq \$ 20,000$ but $<\$ 30,000$ & $22 \%$ \\
\hline$\geq \$ 30,000$ but $<\$ 40,000$ & $9 \%$ \\
\hline$\$ 40,000+$ & $9 \%$ \\
\hline \multicolumn{2}{|l|}{ Marital status } \\
\hline Married/living with partner & $49 \%$ \\
\hline Single & $31 \%$ \\
\hline Divorced & $16 \%$ \\
\hline Separated & $4 \%$ \\
\hline \multicolumn{2}{|l|}{ Children ages 6-18 living with you? } \\
\hline Yes & $71 \%$ \\
\hline No & $29 \%$ \\
\hline \multicolumn{2}{|l|}{ Children ages 5 or younger living with you? } \\
\hline Yes & $38 \%$ \\
\hline No & $62 \%$ \\
\hline
\end{tabular}

reported for continuous variables and percentages for categorical variables. Using longitudinal regression models implemented with Generalized Estimating Equations (GEEs) with robust standard errors, we tested the association between baseline BMI and mean minutes/week of physical activity at followup (months 3, 6). Similarly, we tested whether baseline BMI was associated with mean process variable outcomes at followup. Models adjusted for baseline values of the outcome (self efficacy, cognitive and behavioral strategies, social support) and time, as well as demographics significantly correlated with baseline BMI (education and number of young children in the home). We assumed an autoregressive working correlation matrix. When monthly outcomes data was not available (for Social Support Measures which were measured at baseline and 6 months), we used a linear regression model to test the association between BMI and change in outcome over 6 months, controlling for 
TABLE 2: Effects of time and baseline BMI use on targeted theoretical constructs over time $(n=45)$.

\begin{tabular}{|c|c|c|c|c|}
\hline & $\begin{array}{l}\text { Parameter } \\
\text { Estimate }\end{array}$ & Standard Error & $Z$ & $P$ value \\
\hline \multicolumn{5}{|l|}{ Model 1: self-efficacy } \\
\hline Month & 0.06 & 0.03 & 2.17 & 0.03 \\
\hline Baseline BMI & -0.05 & 0.02 & -2.75 & 0.006 \\
\hline \multicolumn{5}{|l|}{ Model 2: cognitive processes } \\
\hline Month & 0.01 & 0.02 & 0.58 & 0.57 \\
\hline Baseline BMI & -0.04 & 0.02 & -1.97 & 0.05 \\
\hline \multicolumn{5}{|l|}{ Model 3: behavioral processes } \\
\hline Month & 0.04 & 0.02 & 2.35 & 0.02 \\
\hline Baseline BMI & -0.04 & 0.01 & -2.62 & 0.009 \\
\hline Model 4: social support (Family) baseline BMI & -0.89 & 0.33 & -2.74 & 0.009 \\
\hline
\end{tabular}

* Model adjusts for baseline value of outcome, education and number of young children.

baseline education and number of young children in the home. All analyses were carried out using SAS 9.2 and were run on the intent-to-treat sample of $N=45$ participants randomized to the physical activity intervention arm. Eightyseven percent $(N=36)$ of participants returned for the postintervention evaluation. Results were compared to a simple imputation scheme (baseline value carried forward).

\section{Results}

3.1. Demographic Characteristics. The sample was comprised of mostly overweight $(38 \%)$ or obese $(42 \%)$ Latinas with a mean $\mathrm{BMI}$ of $29.32 \mathrm{~kg} / \mathrm{m}^{2}(\mathrm{SD}=4.71)$. Mean age was 41 years old $(\mathrm{SD}=11.18)$ and $80 \%$ reported a household income of less than $\$ 30,000$ per year. Most of the women were married (49\%), unemployed (44\%) and had a high school education or less $(47 \%)$. Ninety-one percent were born outside of the Continental U.S and primarily immigrants from the Dominican Republic, Colombia, Puerto Rico, and Guatemala. See Table 1 for complete demographic information.

3.2. Association between BMI and Physical Activity. Results suggested a trend between baseline BMI and mean minutes/week of moderate intensity or greater physical activity at followup $(b=-5.64$, se $=3.39, P=0.09)$, after adjusting for time, baseline activity, education and number of young children in the home (see Table 2). More specifically, in response to the same 6-month physical activity intervention, someone with a $25 \mathrm{~kg} / \mathrm{m}^{2}$ BMI would report, on average, 27.5 more minutes/week of activity compared to someone with a $30 \mathrm{~kg} / \mathrm{m}^{2}$ BMI at followup.

3.3. Association between BMI and Targeted Theoretical Constructs. Results showed a significant negative association between baseline BMI and mean self-efficacy, cognitive processes, and behavioral processes at any given month $(P<$ $0.05)$. Specifically, participants with higher baseline BMI report lower mean scores on self-efficacy $(b=-0.05$, se $=$ $0.02, P<0.01)$, behavioral processes $(b=-0.04$, se $=0.01$,
$P<0.01)$, and cognitive processes $(b=-0.04$, se $=0.02$, $P=0.04)$ at any given month. In addition, there was a significant association between baseline BMI and change in social support (family) from baseline to 6 months ( $b=$ -0.89 , se $=0.33, P<0.01)$. No significant association was found for the other measures of social support (friends, rewards, and punishments). Results have been summarized in Table 2.

\section{Discussion}

The obesity epidemic has disproportionately impacted Latinas, and adoption of regular physical activity is a known way to help prevent weight gain $[25,26]$. It is important to assess whether our physical activity interventions are successful for all women regardless of BMI and meet the needs of those most at risk for obesity-related health disorders. Higher BMI was associated with lower average change in minutes of at least moderate intensity physical activity at followup. While statistically only a trend was found, the results suggest that, in response to the same 6-month physical activity intervention, someone with a BMI of $25 \mathrm{~kg} / \mathrm{m}^{2}$ reported increasing their average physical activity by about 27.5 more minutes per week than someone with a BMI of $30 \mathrm{~kg} / \mathrm{m}^{2}$. This finding suggests that physical activity interventions may need to be augmented for individuals with higher BMIs to increase their success in changing their level of physical activity.

When examining the process variables that may underlie the changes in activity level, there was a significant relationship between baseline BMI and self-efficacy, behavioral and cognitive processes, and family social support, but not for social support from friends or rewards and punishments. These findings suggest that physical activity interventions for Spanish-speaking Latinas with higher BMIs may need to have additional focus on improving these behavioral processes in order to increase their effectiveness. For example, obese individuals may not feel as confident about their ability to participate in physical activity under certain conditions and may benefit from messages specifically addressing these concerns. Also, certain family dynamics or norms, perhaps 
even disapproval of physical activity by family or other obese family members who are not encouraging or supportive of physical activity, may serve as additional barriers and require direct intervention and/or participation of the full family unit in the program for optimal efficacy. Limitations to the current study include use of a small convenience sample and lack of objective data for physical activity.

In sum, findings from the current study suggest that participants with higher BMIs may need additional intervention to promote physical activity. Future directions should include larger studies to corroborate these findings regarding the influence of BMI on potential intervention efficacy (e.g., changes to physical activity and related process variables) among Latinas. If validated, qualitative research involving focus groups and in-depth individual interviews could help pinpoint what needs are not being met, solicit suggestions on how best to address these barriers, and improve the efficacy of future physical activity programs for individuals with higher BMIs. Such research would be particularly relevant to Latinas due the high prevalence of obesity and related health disparities in this group.

\section{References}

[1] National Center for Health Statistics, Health, United States, 2009 with Chartbook on Trends in the Health of Americans, Hyattsville, Md, USA, 2009.

[2] M. Rosal, B. Olendzki, G. Reed, I. Ockene, O. Gumieniak, and J. Scavron, "Diabetes self-management among low-income Spanish-speaking patients: a pilot study," Annals of Behavioral Medicine, vol. 29, no. 3, pp. 225-235, 2005.

[3] A. C. Lindsay, K. M. Sussner, M. L. Greaney, and K. E. Peterson, "Influence of social context on eating, physical activity, and sedentary behaviors of Latina mothers and their preschool-age children," Health Education and Behavior, vol. 36, no. 1, pp. 81-96, 2009.

[4] National Center for Health Statistics, Health, United States, 2007, with Chartbook on Trends in the Health of Americans, Hyattsville, Md, USA, 2007.

[5] M. Lahti-Koski, P. Pietinen, M. Heliovaara, and E. Vartiainen, "Associations of body mass index and obesity with physical activity, food choices, alcohol intake, and smoking in the 19821997 FINRISK studies," American Journal of Clinical Nutrition, vol. 75, no. 5, pp. 809-817, 2002.

[6] D. Riebe, B. J. Blissmer, M. L. Greaney, C. E. Garber, F. D. Lees, and P. G. Clark, "The relationship between obesity, physical activity, and physical function in older adults," Journal of Aging and Health, vol. 21, no. 8, pp. 1159-1178, 2009.

[7] J. O. Prochaska and C. C. DiClemente, "Stages and processes of self-change of smoking: toward an integrative model of change," Journal of Consulting and Clinical Psychology, vol. 51, no. 3, pp. 390-395, 1983.

[8] A. Bandura, Social Foundations of Thought and Action: A Social Cognitive Theory, Prentice-Hall, Englewood Cliffs, NJ, USA, 1986.

[9] D. W. Pekmezi, C. J. Neighbors, C. S. Lee et al., "A culturally adapted physical activity intervention for Latinas: a randomized controlled trial," American Journal of Preventive Medicine, vol. 37, no. 6, pp. 495-500, 2009.

[10] B. H. Marcus, B. C. Bock, B. M. Pinto, L. H. Forsyth, M. B. Roberts, and R. M. Traficante, "Efficacy of an individualized, motivationally-tailored physical activity intervention," Annals of Behavioral Medicine, vol. 20, no. 3, pp. 174-180, 1998.

[11] B. H. Marcus, M. A. Napolitano, A. C. King et al., "Telephone versus print delivery of an individualized motivationally tailored physical activity intervention: project STRIDE," Health Psychology, vol. 26, no. 4, pp. 401-409, 2007.

[12] B. H. Marcus, B. A. Lewis, D. M. Williams et al., "A comparison of internet and print-based physical activity interventions," Archives of Internal Medicine, vol. 167, no. 9, pp. 944-949, 2007.

[13] B. H. Marcus, K. M. Emmons, L. R. Simkin-Silverman et al., "Evaluation of motivationally tailored vs. Standard self-help physical activity interventions at the workplace," American Journal of Health Promotion, vol. 12, no. 4, pp. 246-253, 1998.

[14] S. N. Blair, W. L. Haskell, and P. Ho, "Assessment of habitual physical activity by a seven-day recall in a community survey and controlled experiments," American Journal of Epidemiology, vol. 122, no. 5, pp. 794-804, 1985.

[15] J. F. Sallis, W. L. Haskell, P. D. Wood et al., "Physical activity assessment methodology in the five-city project," American Journal of Epidemiology, vol. 121, no. 1, pp. 91-106, 1985.

[16] H. A. Hayden-Wade, K. J. Coleman, J. F. Sallis, and C. Armstrong, "Validation of the telephone and in-person interview versions of the 7-day PAR," Medicine and Science in Sports and Exercise, vol. 35, no. 5, pp. 801-809, 2003.

[17] M. A. Pereira, S. J. FitzerGerald, E. W. Gregg et al., "A collection of physical activity questionnaires for health-related research," Medicine \& Science in Sports \& Exercise, vol. 29, no. 6, supplement, pp. S1-S205, 1997.

[18] S. A. Prince, K. B. Adamo, M. E. Hamel, J. Hardt, S. C. Gorber, and M. Tremblay, "A comparison of direct versus self-report measures for assessing physical activity in adults: a systematic review," International Journal of Behavioral Nutrition and Physical Activity, vol. 5, article 55, 2008.

[19] R. Sloane, D. C. Snyder, W. Demark-Wahnefried, D. Lobach, and W. E. Kraus, "Comparing the 7-day physical activity recall with a triaxial accelerometer for measuring time in exercise," Medicine and Science in Sports and Exercise, vol. 41, no. 6, pp. 1334-1340, 2009.

[20] A. L. Dunn, R. E. Andersen, and J. M. Jakicic, "Lifestyle physical activity interventions history, short- and long-term effects, and recommendations," American Journal of Preventive Medicine, vol. 15, no. 4, pp. 398-412, 1998.

[21] A. L. Dunn, B. H. Marcus, J. B. Kampert, M. E. Garcia, H. W. Kohl, and S. N. Blair, "Comparison of lifestyle and structured interventions to increase physical activity and cardiorespiratory fitness: a randomized trial," Journal of the American Medical Association, vol. 281, no. 4, pp. 327-334, 1999.

[22] B. H. Marcus, V. C. Selby, R. S. Niaura, and J. S. Rossi, "Selfefficacy and the stages of exercise behavior change," Research Quarterly for Exercise and Sport, vol. 63, no. 1, pp. 60-66, 1992.

[23] B. H. Marcus, J. S. Rossi, V. C. Selby, R. S. Niaura, and D. B. Abrams, "The stages and processes of exercise adoption and maintenance in a worksite sample," Health Psychology, vol. 11, no. 6, pp. 386-395, 1992.

[24] J. F. Sallis, R. M. Grossman, R. B. Pinski, T. L. Patterson, and P. R. Nader, "The development of scales to measure social support for diet and exercise behaviors," Preventive Medicine, vol. 16, no. 6, pp. 825-836, 1987.

[25] W. H. Saris, S. N. Blair, M. A. van Baak et al., "How much physical activity is enough to prevent unhealthy weight gain? Outcome of the IASO 1st stock conference and consensus statement," Obesity Reviews, vol. 4, no. 2, pp. 101-114, 2003. 
[26] V. A. Catenacci and H. R. Wyatt, "The role of physical activity in producing and maintaining weight loss," Nature Clinical Practice Endocrinology and Metabolism, vol. 3, no. 7, pp. 518529, 2007. 


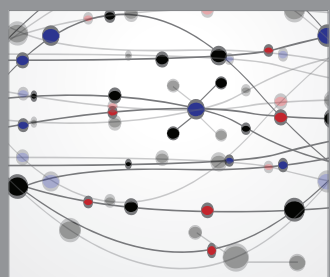

The Scientific World Journal
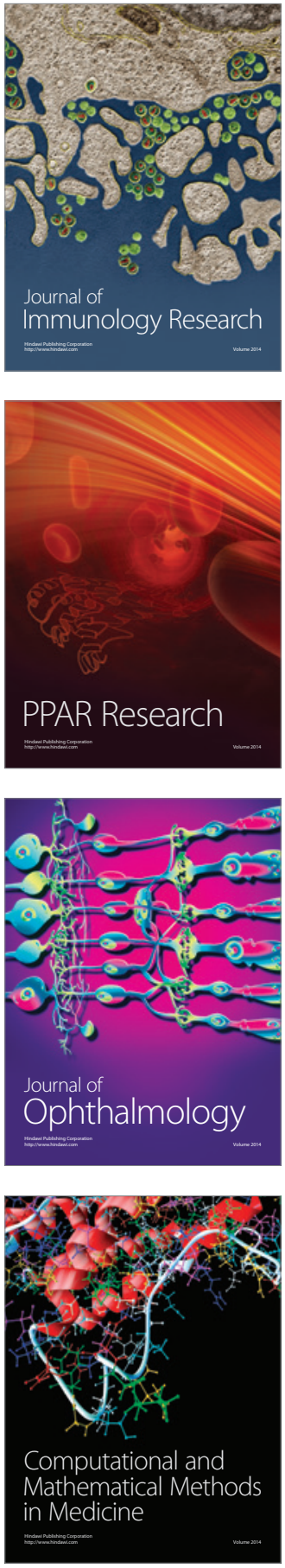

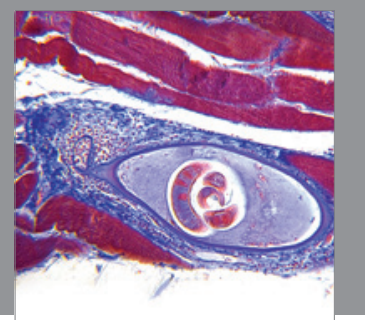

Gastroenterology

Research and Practice
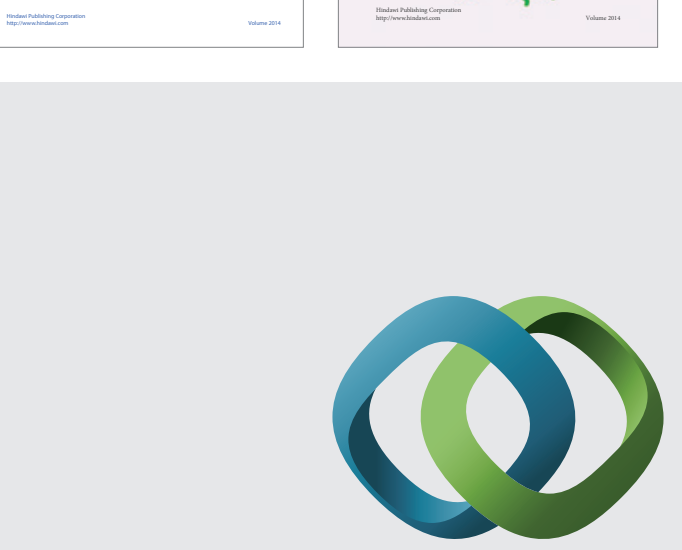

\section{Hindawi}

Submit your manuscripts at

http://www.hindawi.com
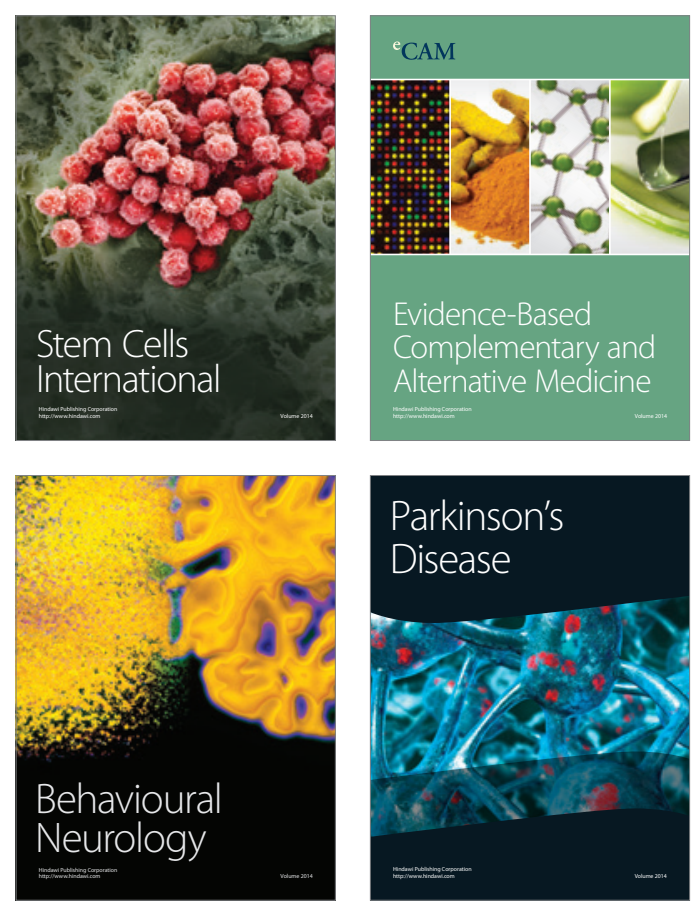

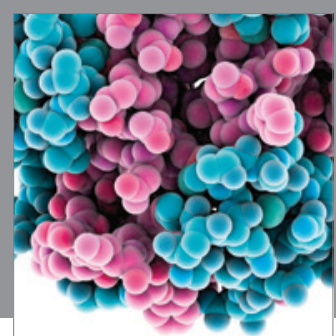

Journal of
Diabetes Research

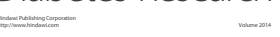

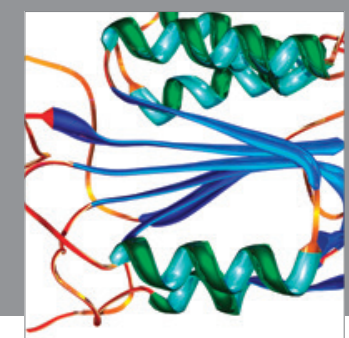

Disease Markers
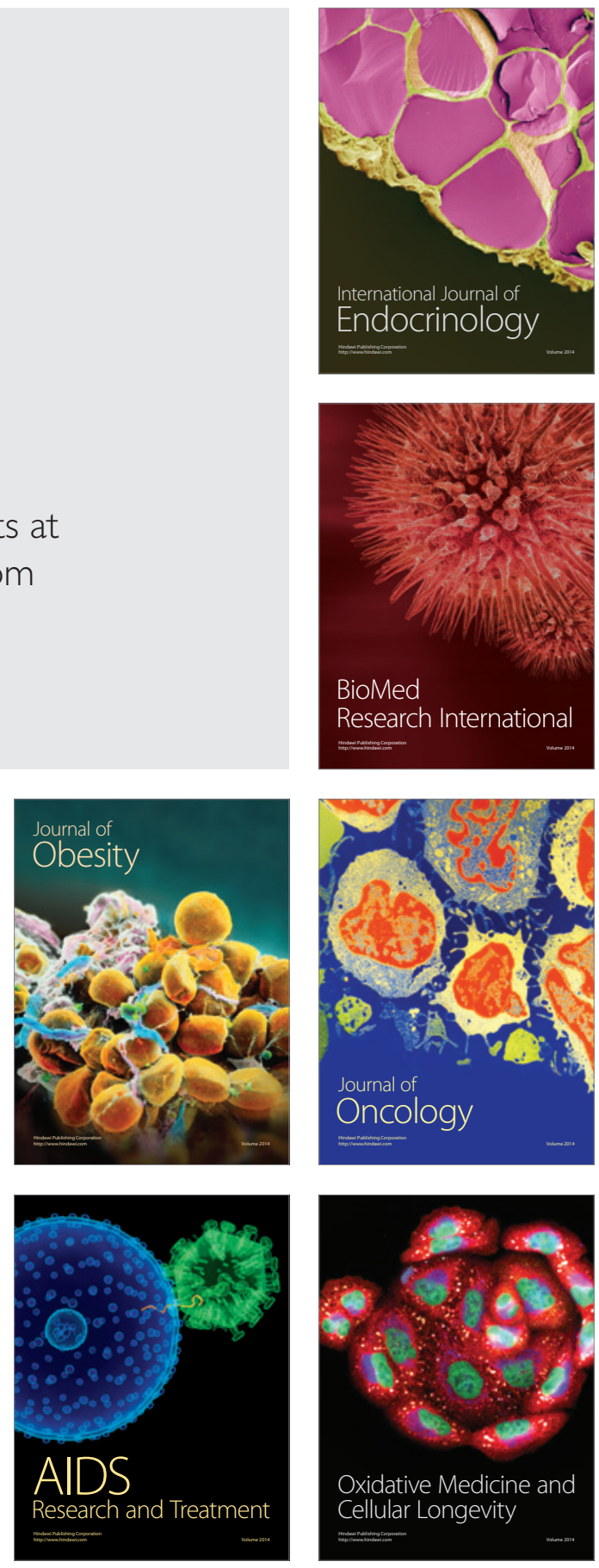